\title{
Hearing and dementia
}

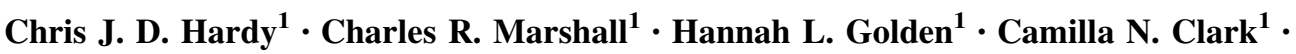 \\ Catherine J. Mummery $^{1,4}$ - Timothy D. Griffiths ${ }^{5,6}$ - Doris-Eva Bamiou ${ }^{2,3,6}$. \\ Jason D. Warren ${ }^{1,6}$
}

Received: 27 April 2016/Revised: 13 June 2016/Accepted: 14 June 2016/Published online: 2 July 2016

(C) The Author(s) 2016. This article is published with open access at Springerlink.com

\begin{abstract}
Hearing deficits associated with cognitive impairment have attracted much recent interest, motivated by emerging evidence that impaired hearing is a risk factor for cognitive decline. However, dementia and hearing impairment present immense challenges in their own right, and their intersection in the auditory brain remains poorly understood and difficult to assess. Here, we outline a clinically oriented, symptom-based approach to the assessment of hearing in dementias, informed by recent progress in the clinical auditory neuroscience of these diseases. We consider the significance and interpretation of hearing loss and symptoms that point to a disorder of auditory cognition in patients with dementia. We identify key auditory characteristics of some important dementias
\end{abstract}

Jason D. Warren

jason.warren@ucl.ac.uk

1 Department of Neurodegenerative Disease, Dementia Research Centre, UCL Institute of Neurology, University College London, Queen Square, London WC1N 3BG, UK

2 Department of Neuro-otology, National Hospital for Neurology and Neurosurgery, Queen Square, London, UK

3 UCL Ear Institute, University College London, London, UK

4 Cognitive Disorders Clinic for the Deaf, National Hospital for Neurology and Neurosurgery, Queen Square, London, UK

5 Auditory Group, Institute of Neuroscience, The Medical School, University of Newcastle upon Tyne, Newcastle upon Tyne, UK

6 Central Auditory Disorders Clinic, National Hospital for Neurology and Neurosurgery, Queen Square, London, UK and conclude with a bedside approach to assessing and managing auditory dysfunction in dementia.

Keywords Hearing · Auditory · Dementia - Alzheimer's disease - Frontotemporal dementia · Progressive aphasia . Lewy body disease

\section{Introduction}

Although hearing impairment is not generally regarded as a cardinal feature of dementia, hearing in patients with dementia is a focus of growing clinical interest. Recent evidence suggests that hearing loss may predict or accelerate cognitive deterioration [1-3], and alterations of hearing may manifest as complex cognitive and behavioural symptoms relevant to the differential diagnosis of dementias [4-10]. Interventions targeting auditory processes (most notably, music) have gained wide currency $[4,11]$. However, the organisation of the human auditory brain is complex and incompletely understood. Moreover, neuropsychological frameworks for characterising hearing disorders produced by brain disease and practical tools for assessing auditory functions suitable for use in cognitively impaired patients are often lacking.

In this review, we outline a clinically oriented, symptom-based approach to hearing in dementia, informed by recent progress in the clinical auditory neuroscience of these diseases. We consider the problem of hearing loss (impaired detection of sound and how this interacts with cognitive function) and symptoms that point to a disorder of auditory cognition (impaired understanding or behavioural responses to sound). We identify key auditory characteristics of some important dementias. We conclude 
with a bedside approach to assessing and managing auditory dysfunction in dementia.

\section{The auditory brain and dementia}

\section{Neuropsychology of hearing}

Hearing (considered broadly as the function of the human auditory brain and its peripheral end organs) has been aligned with other complex neuropsychological processes based on studies of the normal brain and focal brain damage [12, 13]. Together, this evidence suggests a hierarchical organisation that differentiates categories and stages of auditory information processing (Table 1). Processing of sound begins in the ascending auditory pathways extending from cochlea to primary auditory cortex in Heschl's gyrus: this is not a passive relay but involves considerable signal transformation [12]. While the terminology of hearing disorders is problematic, in consideration of disease associations, it is useful to attempt to distinguish between peripheral (predominantly cochlear or auditory nerve), subcortical (ascending auditory pathway), and cortical auditory dysfunction. Auditory cognition-processing beyond sound detection leading to auditory perception and understanding-is mediated by distributed networks involving auditory cortex and its cerebral connections; disorders affecting these networks produce characteristic symptoms and syndromes of auditory cognitive dysfunction (summarised in Table 1).

As a framework for analysing disorders of auditory cognition, it is useful to consider complex sounds (speech, voices, music, and environmental noises) as 'auditory objects' that must be disambiguated from the auditory background and organised into coherent perceptual representations [13]. The processing of such sound objects entails perceptual analysis (encoding of acoustic features, such as pitch, rhythm, and timbre) leading to semantic processing (extraction of associated meaning, leading to sound recognition) $[13,14]$. In the world at large, sounds are embedded in auditory scenes that must be actively deconstructed to identify and track sounds of interest [10]: this, in turn, requires the representation of sound location and movement (auditory spatial analysis) and abstraction of identifying sound characteristics under varying listening conditions (auditory apperceptive processing). Many sounds also have emotional and behavioural relevance.

\section{The burden of dementia}

Dementia is arguably the most significant public health problem confronting ageing societies, with an estimated 800,000 sufferers currently in the United Kingdom alone
[15]. However, 'dementia' designates a syndrome of acquired, progressive, socially and/or occupationally significant cognitive and/or behavioural decline: this definition embraces over a hundred highly diverse diseases, the most common of which is Alzheimer's disease (AD) [4, 16-18]. Here, we focus on major neurodegenerative causes of dementia in mid to later life, collectively characterised by pathogenic protein spread over large-scale cerebral networks and distinctive profiles of regional brain atrophy and clinical deficits (summarised in Table 2). Brain networks targeted by these diseases overlap the temporal, parietal, frontal, and subcortical circuitry that supports auditory cognition (Tables 1,2 ): this is key to anticipating and understanding the disorders of hearing that accompany particular dementia syndromes.

\section{Hearing loss and dementia}

\section{Epidemiological evidence}

Significant hearing loss (operationally, $>20 \mathrm{~dB}$ elevation of threshold for pure tone detection) affects around $40 \%$ of those aged over 65 [19] and has important links to cognitive impairment and dementia. Age-related hearing loss (presbycusis) commonly results from cochlear dysfunction, though age-related alterations in more central auditory pathways may also be relevant and have probably been under-recognised [20]. The balance of epidemiological evidence across populations suggests that hearing loss is associated with cognitive decline and constitutes a risk factor for development of dementia in older adults, though the strength of this association is somewhat variable [20, 21]. One meta-analysis concluded that cognitive and hearing impairment are correlated and that hearing loss impacts on multiple domains of cognition [21]; this is not simply attributable to hearing loss confounding speech-based cognitive tasks [20] and has been observed in those with and without dementia [22]. Hearing loss $\sim 25 \mathrm{~dB}$ has an effect on cognitive deterioration equivalent to around 7 years of ageing [1] and risk of dementia increases with increasing severity of hearing impairment [2].

\section{The role of peripheral hearing}

While the association between hearing loss and cognitive decline appears robust, the mechanism remains unresolved. Hearing impairment might accelerate cognitive decline by compounding sensory and social isolation, increasing cognitive load, and thereby exhausting compensatory cognitive reallocation, or constitute a nonspecific marker of frailty [20]. However, the association 
Table 1 An outline of core operations in auditory cognition and their clinical and neuroanatomical correlates

\begin{tabular}{|c|c|c|c|c|}
\hline $\begin{array}{l}\text { Auditory } \\
\text { cognitive } \\
\text { operation }\end{array}$ & Clinical correlates & $\begin{array}{l}\text { Neuropsychological } \\
\text { tests }\end{array}$ & Procedure $^{\mathrm{a}}$ & $\begin{array}{l}\text { Neuroanatomical } \\
\text { correlates }[13,82]\end{array}$ \\
\hline Feature detection & $\begin{array}{l}\text { Cortical deafness }{ }^{\mathrm{b}}, \\
\text { tinnitus }^{\mathrm{c}}\end{array}$ & $\begin{array}{l}\text { Sound detection } \\
\text { Gap-in-noise detection } \\
\text { AM/FM }{ }^{d} \text { detection } \\
\text { Spatial lateralisation }\end{array}$ & $\begin{array}{l}\text { Detection of any sound (e.g., tone) behaviourally/EP [83] } \\
\text { Detection of short silent interval in white noise burst [76] } \\
\text { Detection of modulation (vibrato) of intensity/pitch in carrier } \\
\text { tone [76] } \\
\text { Detection of right-left sound shift based on inter-aural phase/ } \\
\text { intensity cues [76] }\end{array}$ & $\begin{array}{l}\text { PAC, lat HG, PT, } \\
\text { pSTG, subcortical } \\
\text { circuits }\end{array}$ \\
\hline Feature analysis & $\begin{array}{l}\text { Word deafness }{ }^{\mathrm{e}}, \\
\text { dystimbria }^{\mathrm{f}}, \text { amusia }^{\mathrm{g}}\end{array}$ & $\begin{array}{l}\text { Phoneme } \\
\text { discrimination } \\
\text { MBEA pitch/temporal } \\
\text { subtests }\end{array}$ & $\begin{array}{l}\text { Discrimination of sound pairs/sequences differing in pitch, } \\
\text { temporal or timbral characteristics }[49,79,84] \\
\text { Labelling of features in a single sound (e.g., tone glide } \\
\text { direction 'up' or 'down') [7] }\end{array}$ & $\begin{array}{l}\text { lat HG, pSTG/STS, } \\
\text { aSTG, subcortical } \\
\text { circuits }\end{array}$ \\
\hline Scene analysis & Auditory disorientation & $\begin{array}{l}\text { SSI-ICM } \\
\text { Speech-in-noise }^{\mathrm{h}} \\
\text { Spatial localisation } \\
\text { Dichotic listening }\end{array}$ & $\begin{array}{l}\text { Identification of a sentence spoken over background message } \\
\text { same ear [34] } \\
\text { Identification of words against background noise/multi-talker } \\
\text { babble } \\
\text { Discrimination of sound location/movement in real or virtual } \\
\text { space [9, 10] } \\
\text { Attention to one of two stimuli played simultaneously via each } \\
\text { ear [23] }\end{array}$ & $\begin{array}{l}\text { PT/pSTG, IPL, PFC, } \\
\text { hippocampus, } \\
\text { subcortical circuits }\end{array}$ \\
\hline $\begin{array}{l}\text { Object } \\
\text { representation } \\
\text { (apperceptive } \\
\text { processing) }\end{array}$ & $\begin{array}{l}\text { Auditory apperceptive } \\
\text { agnosias, musical and } \\
\text { verbal hallucinations }\end{array}$ & $\begin{array}{l}\text { Melody discrimination } \\
\text { Distorted melodies } \\
\text { Voice discrimination } \\
\text { Accent processing }\end{array}$ & $\begin{array}{l}\text { Discrimination of (unfamiliar) melodies }[56,85] \\
\text { Identification of an altered familiar melody }[85] \\
\text { Discrimination of (unfamiliar) speakers [57] } \\
\text { Speech perception under unfamiliar accents }[8,52]\end{array}$ & $\begin{array}{l}\text { PT, pSTG/STS, IPL, } \\
\text { aSTG }\end{array}$ \\
\hline $\begin{array}{l}\text { Object } \\
\text { recognition } \\
\text { (semantic } \\
\text { processing) }\end{array}$ & $\begin{array}{l}\text { Auditory associative } \\
\text { agnosias (including } \\
\text { phonagnosia) }\end{array}$ & $\begin{array}{l}\text { Environmental sound, } \\
\text { melody, voice } \\
\text { recognition }\end{array}$ & $\begin{array}{l}\text { Recognition of familiar sounds, tunes, voices; conventionally } \\
\text { assessed by naming the target but can be assessed by forced- } \\
\text { choice or matching cross-modally (e.g., sound-picture) [56] } \\
\text { or within-modality (perceptually dissimilar sound excerpts, } \\
\text { categorisation based on semantic characteristic) } \\
{[7,49,54,55] \text {, familiarity decision [56] in patients with }} \\
\text { aphasia }\end{array}$ & aSTG, TP, insula \\
\hline $\begin{array}{l}\text { Emotional } \\
\text { valuation }\end{array}$ & $\begin{array}{l}\text { Receptive dysprosodia, } \\
\text { auditory anhedonia, } \\
\text { Musicophilia }\end{array}$ & $\begin{array}{l}\text { Emotion recognition } \\
\text { Emotional response }\end{array}$ & $\begin{array}{l}\text { Naming, forced choice }[55,64,66,86] \text { or cross-modal } \\
\text { labelling of emotions in sounds } \\
\text { Behavioural rating of valence, arousal; autonomic indices [68] }\end{array}$ & $\begin{array}{l}\text { MTL, insula, OFC, } \\
\text { ACC, mesolimbic/ } \\
\text { striatal circuits }\end{array}$ \\
\hline $\begin{array}{l}\text { Working memory/ } \\
\text { attention }^{j}\end{array}$ & $\begin{array}{l}\text { Auditory neglect/ } \\
\text { inattention }\end{array}$ & $\begin{array}{l}\text { Compare sequential } \\
\text { sounds } \\
\text { Oddball detection } \\
\text { Dichotic listening }\end{array}$ & $\begin{array}{l}n \text {-back tasks (e.g., [77]) } \\
\text { Sustained attention with detection of target deviants } \\
\text { behaviourally/EP [87] } \\
\text { Attention to one of two stimuli played simultaneously via each } \\
\text { ear [75] }\end{array}$ & $\begin{array}{l}\text { Fronto-parieto- } \\
\text { temporal, } \\
\text { subcortical circuits }\end{array}$ \\
\hline
\end{tabular}

$A C C$ anterior cingulate cortex, $A M / F M$ amplitude/frequency modulation, a/pSTG anterior/posterior superior temporal gyrus, $E P$ electrophysiological evoked potentials, IPL inferior parietal lobe, lat HG lateral Heschl's gyrus, MBEA Montreal Battery for Evaluation of Amusia, MTL mesial temporal lobe, $O F C$ orbitofrontal cortex, $P A C$ primary auditory cortex, $P F C$ prefrontal cortex, $P T$ planum temporale, SSI-ICM synthetic sentence identification with ipsilateral competing message, STS superior temporal sulcus, TP temporal pole

${ }^{a}$ Few widely available tests or population norms are available for auditory cognition. These are mainly used in research settings, but certain instruments may be suitable for systematic clinical assessment of cognitively impaired patients (e.g., Newcastle Auditory Battery [76]; Montreal Battery for Evaluation of Amusia [77]; Queen Square Tests of Auditory Cognition for auditory object processing, voice and scene analysis $[6-9,48,57])$

b Subtotal cortical deafness often manifests as auditory agnosia

c Tinnitus is mediated by distributed circuitry also including subcortical, anterior and limbic structures

${ }^{\mathrm{d}}$ Neuroanatomical correlates vary with modulation rate

e Mechanism of word deafness may be heterogeneous

${ }^{\mathrm{f}}$ Impaired perception of timbre (that property distinguishing two sounds of identical pitch, duration and loudness, e.g., musical instrument voices)

g Impaired perception of music due to a cerebral cause

h Speech-in-noise perception is impaired with cochlear dysfunction so interpretation of any more central deficit must be cautious [23]

${ }^{\mathrm{i}}$ Maintaining alertness and attention

${ }^{\mathrm{j}}$ Particularly during auditory scene analysis but relevant to auditory sequence processing more generally (auditory neglect/inattention unusual in dementia but impaired monitoring of acoustic events common) 
Table 2 Summary of major neurodegenerative dementias, emphasising auditory characteristics

\begin{tabular}{|c|c|c|c|c|c|c|c|c|}
\hline \multirow[t]{2}{*}{ Disease/syndrome } & \multirow{2}{*}{$\begin{array}{l}\text { Core clinical } \\
\text { phenotype }\end{array}$} & \multirow{2}{*}{$\begin{array}{l}\text { Key auditory } \\
\text { symptoms }\end{array}$} & \multicolumn{5}{|c|}{ Auditory cognitive processes ${ }^{a}$} & \multirow{2}{*}{$\begin{array}{l}\text { Pathological } \\
\text { neuroanatomy }\end{array}$} \\
\hline & & & Perc & App & Sem & Em & Wm/Att & \\
\hline AD: typical $[6-10,57,64,88-94]$ & $\begin{array}{l}\text { Episodic, } \\
\text { topographical } \\
\text { memory loss, } \\
\text { parietal deficits }\end{array}$ & \multirow{3}{*}{$\begin{array}{l}\text { Difficulty tracking } \\
\text { sound objects } \\
\text { and information } \\
\text { in busy acoustic } \\
\text { environments, } \\
\text { auditory } \\
\text { disorientation, } \\
\text { increased sound } \\
\text { sensitivity }\end{array}$} & + & ++ & + & - & ++ & $\begin{array}{l}\text { PCC, precuneus, } \\
\text { temporo-parietal } \\
\text { cortices }\end{array}$ \\
\hline$P C A^{\mathrm{c}}[9]$ & $\begin{array}{l}\text { Visuo-perceptual, } \\
\text { visuo-spatial, } \\
\text { other parietal } \\
\text { deficits }\end{array}$ & & + & + & & & & \\
\hline$L P A^{\mathrm{c}}[7,48,50,64]$ & $\begin{array}{l}\text { Anomia, } \\
\text { phonemic and } \\
\text { verbal working } \\
\text { memory deficits }\end{array}$ & & - & + & + & + & ++ & \\
\hline${\mathrm{PDD} / \mathrm{DLB}^{\mathrm{d}}}[59,60,87,95]$ & $\begin{array}{l}\text { Fluctuating } \\
\text { executive, } \\
\text { attentional } \\
\text { deficits, } \\
\text { bradyphrenia, } \\
\text { visual } \\
\text { hallucinations, } \\
\text { parkinsonism }\end{array}$ & $\begin{array}{l}\text { Auditory } \\
\text { hallucinations }\end{array}$ & + & & & + & + & $\begin{array}{l}\text { Cortico-subcortical } \\
\text { circuits }\end{array}$ \\
\hline \multicolumn{9}{|l|}{ FTLD: sporadic/undefined } \\
\hline$b v F T D[51,54,68,96,97]$ & $\begin{array}{l}\text { Socio-emotional, } \\
\text { executive } \\
\text { dysfunction with } \\
\text { disinhibition, } \\
\text { apathy, } \\
\text { obsessionality, } \\
\text { other } \\
\text { behavioural } \\
\text { abnormalities }\end{array}$ & $\begin{array}{l}\text { Sound aversion, } \\
\text { phonagnosia, } \\
\text { altered attentive } \\
\text { processing of } \\
\text { auditory stimuli }\end{array}$ & - & - & + & ++ & ++ & $\begin{array}{l}\text { Auditory and } \\
\text { multimodal } \\
\text { association cortex } \\
\text { in ant TL, OFC, } \\
\text { insula, ACC, striatal } \\
\text { circuits }\end{array}$ \\
\hline $\begin{array}{l}S D \\
\quad[5,48,49,51,53,54,56,57,68,98]\end{array}$ & $\begin{array}{l}\text { Vocabulary loss, } \\
\text { visual agnosia } \\
\text { due to impaired } \\
\text { semantic } \\
\text { memory, } \\
\text { behavioural } \\
\text { changes similar } \\
\text { to bvFTD }\end{array}$ & $\begin{array}{l}\text { Musicophilia, } \\
\text { tinnitus; } \\
\text { phonagnosia/ } \\
\text { nonverbal sound } \\
\text { agnosia }\end{array}$ & - & + & ++ & ++ & - & $\begin{array}{l}\text { Auditory/multimodal } \\
\text { association cortex } \\
\text { in ant TL, OFC, } \\
\text { insula }\end{array}$ \\
\hline PNFA $[7,8,48,49,52,64]$ & $\begin{array}{l}\text { Speech production } \\
\text { deficits, } \\
\text { agrammatism }\end{array}$ & $\begin{array}{l}\text { Agnosia for } \\
\text { environmental } \\
\text { sounds, accents, } \\
\text { word deafness }\end{array}$ & ++ & + & + & + & ++ & $\begin{array}{l}\text { Peri-Sylvian } \\
\text { networks }\end{array}$ \\
\hline \multicolumn{9}{|l|}{ FTLD: genetic } \\
\hline MAPT [51, 54, 99] & $\begin{array}{l}\text { Similar bvFTD, } \\
\text { may have } \\
\text { associated } \\
\text { parkinsonism }\end{array}$ & $\begin{array}{l}\text { Altered hedonic } \\
\text { responses to } \\
\text { sound }\end{array}$ & + & & ++ & ++ & & $\begin{array}{l}\text { Ant TL/fronto- } \\
\text { subcortical network }\end{array}$ \\
\hline C9orf72 $[56,68,99,100]$ & $\begin{array}{l}\text { Similar bvFTD or } \\
\text { PNFA, may } \\
\text { have associated } \\
\text { motor neuron } \\
\text { features }\end{array}$ & $\begin{array}{l}\text { Auditory } \\
\text { hallucinations }\end{array}$ & - & & + & + & + & $\begin{array}{l}\text { Cortico-thalamo- } \\
\text { cerebellar network }\end{array}$ \\
\hline$G R N^{\mathrm{e}}[7,64,101,102]$ & $\begin{array}{l}\text { Similar bvFTD or } \\
\text { mixed aphasia, } \\
\text { often prominent } \\
\text { parietal signs }\end{array}$ & $\begin{array}{l}\text { Limited } \\
\text { information }\end{array}$ & + & + & - & - & + & $\begin{array}{l}\text { Distributed intra- } \\
\text { hemispheric } \\
\text { networks }\end{array}$ \\
\hline
\end{tabular}


Table 2 continued

\begin{tabular}{|c|c|c|c|c|c|c|c|c|}
\hline \multirow[t]{2}{*}{ Disease/syndrome } & \multirow{2}{*}{$\begin{array}{l}\text { Core clinical } \\
\text { phenotype }\end{array}$} & \multirow{2}{*}{$\begin{array}{l}\text { Key auditory } \\
\text { symptoms }\end{array}$} & \multicolumn{5}{|c|}{ Auditory cognitive processes ${ }^{a}$} & \multirow{2}{*}{$\begin{array}{l}\text { Pathological } \\
\text { neuroanatomy }\end{array}$} \\
\hline & & & Perc & App & Sem & Em & $\overline{\mathrm{Wm} / \mathrm{Att}}$ & \\
\hline CBS/PSP $[103,104]$ & $\begin{array}{l}\text { Executive deficits, } \\
\text { bradyphrenia in } \\
\text { context } \\
\text { parkinsonism, } \\
\text { supranuclear } \\
\text { gaze palsy, limb } \\
\text { dystonia - } \\
\text { apraxia }\end{array}$ & $\begin{array}{l}\text { Agnosia for } \\
\text { environmental } \\
\text { sounds, } \\
\text { disordered voice } \\
\text { emotion } \\
\text { processing }\end{array}$ & - & & + & + & & $\begin{array}{l}\text { Cortico-subcortical } \\
\text { circuits, IFG }\end{array}$ \\
\hline $\operatorname{HD}^{\mathrm{d}}[105,106]$ & $\begin{array}{l}\text { Executive and } \\
\text { behavioural } \\
\text { changes with } \\
\text { chorea }\end{array}$ & $\begin{array}{l}\text { Attentive } \\
\text { processing of } \\
\text { auditory stimuli }\end{array}$ & + & & & & + & $\begin{array}{l}\text { Cortico-subcortical } \\
\text { circuits }\end{array}$ \\
\hline Prion diseases $[40,44,67]$ & $\begin{array}{l}\text { Usually rapid } \\
\text { global dementia } \\
\text { with prominent } \\
\text { myoclonus, } \\
\text { ataxia; wide } \\
\text { phenotypic } \\
\text { variation } \\
\text { (especially } \\
\text { genetic forms) }\end{array}$ & $\begin{array}{l}\text { Occasionally } \\
\text { tinnitus, cortical } \\
\text { deafness, } \\
\text { auditory } \\
\text { hallucinations, } \\
\text { increased sound } \\
\text { sensitivity }\end{array}$ & + & + & & & & $\begin{array}{l}\text { Primary auditory } \\
\text { cortex }\end{array}$ \\
\hline
\end{tabular}

$A C C$ anterior cingulate cortex, $A D$ Alzheimer's disease, ant $T L$ anterior temporal lobe, App auditory apperception (including parsing of auditory scenes into constituent sound objects), bvFTD behavioural variant frontotemporal dementia, C9orf72 mutations in open reading frame 72 on chromosome 9, CBS/PSP corticobasal syndrome/progressive supranuclear palsy, Ep mem episodic memory for nonverbal sounds (including music), Em emotion processing from sounds (including music/prosody), FTLD frontotemporal lobar degeneration, GRN progranulin gene mutations, $H D$ Huntington's disease, $L P A$ logopenic aphasia, MAPT microtubule-associated protein tau gene mutations, $O F C$ orbitofrontal cortex, $P C A$ posterior cortical atrophy, $P C C$ posterior cingulate cortex, Perc early auditory perception (acoustic feature detection and analysis), $P D D / D L B$ Parkinson's disease/Lewy body dementia, PNFA progressive nonfluent aphasia, $S D$ semantic dementia, Sem semantic processing of sounds (including melodies), Wm/Att nonverbal auditory working memory/attention

+ deficit documented, ++ particularly severe in relation to other deficits, - deficit absent/inconsistent, blank cells indicate no adequate data available

${ }^{a}$ Defined by performance on behavioural tests

b Distribution of pathological changes in brain networks relevant to auditory deficits, as assessed using voxel-based morphometry, functional MRI and/or post mortem material

${ }^{c}$ Underpinned by Alzheimer pathology in $>80 \%$ of cases

d Abnormalities of rhythm processing in basal ganglia and cerebellar degenerations [95, 107]

e Limited information currently for progressive aphasia presentation only

between impaired hearing and cognition remains after controlling for other demographic and comorbidity factors $[2,23]$. Peripheral hearing loss might hasten neurodegenerative processes more directly. Hearing loss in older adults correlates with tissue volume loss in auditory cortex [24], temporal lobe, and whole brain [3], and is associated with functional reorganisation of auditory cortical networks consistent with more effortful listening and reduced cognitive reserve [25]. Though limited histopathological evidence is available concerning the auditory system in common dementias, major auditory relay nuclei are involved pathologically in $\operatorname{AD}[26,27]$, while animal models suggest that peripheral deafferentation disrupts hippocampal function [28, 29].

\section{The role of 'central' auditory processing}

Auditory deficits in AD may be disproportionate to any abnormality of sound detection or otological markers [20, 30-34]: while the neuroanatomical correlates of 'central' hearing measures have not been fully defined, such deficits may reflect disordered cortical mechanisms of auditory scene analysis (Table 1). This is corroborated by other evidence that abnormalities of auditory cortical evoked potentials predate clinical symptoms in young carriers of pathogenic AD mutations [35]. Information for other dementias remains very limited. Relatively, a few studies of hearing in dementia have addressed cortical auditory processing specifically, perhaps partly 
accounting for the wide variation in reported frequency of hearing impairment in $\mathrm{AD}[4,36]$ : an observation that seems otherwise difficult to reconcile with epidemiological data.

The effects of hearing impairment on cognitive decline might be most parsimoniously considered as an interaction of peripheral and more central factors. The auditory system has extensive efferent as well as afferent traffic [12] allowing for reciprocal interaction between cortical, brainstem, and peripheral mechanisms [37]. Moreover, in practice, these can be challenging to disambiguate in individual patients.

\section{Syndromes of dementia and hearing loss}

Syndromic associations of dementia with dysfunction of cochlea or ascending auditory pathways are uncommon and generally occur in the context of more complex neurological impairment, often in younger patients; examples are summarised in Table 3.

\section{Symptoms of altered auditory cognition in dementia}

Though auditory dysfunction is rarely the presenting feature, histopathological involvement of auditory cortices has been described in major neurodegenerative dementias [26, 38-41], and deficits of auditory cognition (Table 1) are not uncommon early features of these diseases. Certain general observations suggest an auditory cognitive disorder: the patient typically experiences greater listening difficulties and derives less benefit with conventional binaural amplification than anticipated from the degree of audiometric loss and may also exhibit various abnormal behavioural responses to sounds. Matching of incoming sound information to stored neural 'templates' based on past experience of the auditory world may be a general operating principle of the auditory brain [14, 42]: disruption of this process with neurodegenerative pathologies may lead to deficient perception or to aberrant perception of sounds. Deficient sound perception or recognition not attributable to faulty peripheral encoding constitutes an auditory agnosia, which may be selective for particular kinds of sounds; aberrant 'excessive' processing may manifest as auditory hallucinations. These disorders of auditory cognition commonly coexist.

Here, we emphasise the differential diagnosis of auditory symptoms; characterisation of auditory deficits using neuropsychological tests is a complementary enterprise. Together, these approaches define auditory phenotypes, summarised for selected dementias in Table 2; neuroanatomical correlates are shown in Fig. 1.

\section{Impaired perception of sound features}

Patients with dementia may have reduced perception of sound disproportionate to any damage involving cochlea or ascending auditory pathways: this may manifest as cortical deafness (described rarely in prion disease: $[43,44]$ ) or relatively selective 'word deafness' or auditory agnosia, more commonly described with progressive nonfluent aphasia and (for unknown reasons) in Japanese patients [45-47]. A useful clinical clue to word deafness is substantially better comprehension of written than spoken language. Speech perception may be particularly vulnerable as it depends on precise temporal feature decoding but may signify a more generic impairment of auditory feature analysis in syndromes with peri-Sylvian degeneration [48, 49].

\section{Impaired perception of auditory scenes and objects}

Frank auditory disorientation is uncommon in dementia though does occur (usually accompanying visual disorientation) in patients with posterior cortical degenerations [50]. However, patients with both posterior cortical atrophy and clinically typical AD commonly report difficulty following conversations and other sounds against background noise, and this may contribute to their avoiding social situations and a general dislike of busy auditory environments [51]. Such symptoms may develop early in the course of the illness and without other evidence of hearing loss and (though often attributed to a nonspecific memory or attentional deficit) may signify $\mathrm{AD}$-associated impairments of auditory scene and auditory spatial analysis, correlated in structural and functional neuroanatomical studies with disintegration of a core parieto-temporal network $[6,9,10]$. Auditory scene analysis depends on accurate parsing of the acoustic stream into constituent sound objects, mediated by sensory computational mechanisms under attentional and executive control; these mechanisms interact in temporoparietal cortical 'hub' regions.

Other symptoms, experienced particularly by patients with $\mathrm{AD}$ and progressive nonfluent aphasia suggest auditory apperceptive dysfunction: disproportionate difficulty identifying or understanding sound objects under unusual or degraded listening conditions. The patient may not recognise a familiar voice over a noisy telephone line or may fail to understand a message delivered in an unfamiliar accent $[8,52])$. Such symptoms may have a neuroanatomical substrate in posterior peri-Sylvian cortices, similar to that underpinning impaired auditory scene analysis.

\section{Impaired recognition of sounds}

Patients with semantic dementia develop deficits of nonverbal sound recognition (auditory associative agnosia) as 
Table 3 Some syndromes with peripheral or subcortical hearing impairment and dementia

\begin{tabular}{|c|c|c|c|c|}
\hline Disease & Aud & Cogn & Associated features & Diagnostic investigations \\
\hline \multicolumn{5}{|l|}{ Inflammatory } \\
\hline $\begin{array}{l}\text { Antiphospholipid } \\
\text { syndrome [108] }\end{array}$ & F; C, & $\mathrm{F}$ & $\begin{array}{l}\text { Headache, seizures, chorea, myelopathy, optic } \\
\text { neuritis, vestibulopathy }\end{array}$ & $\begin{array}{l}\text { Antibody profile with compatible clinical } \\
\text { phenotype }\end{array}$ \\
\hline $\begin{array}{l}\text { Multiple sclerosis } \\
\text { [109] }\end{array}$ & $\mathrm{U} ; \mathrm{RC}^{\mathrm{a}}$ & $\mathrm{F}^{\mathrm{b}}$ & $\begin{array}{l}\text { Diverse: vertigo, optic neuritis, various brainstem, } \\
\text { cerebral, spinal signs }\end{array}$ & $\begin{array}{l}\text { Compatible clinical and MRI features of CNS } \\
\text { demyelination, (McDonald criteria), supported } \\
\text { by CSF unmatched oligoclonal bands }\end{array}$ \\
\hline $\begin{array}{l}\text { Neuro-Behçet's } \\
\text { [110] }\end{array}$ & $\mathrm{F} ; \mathrm{RC}^{\mathrm{a}}$ & $\mathrm{U}^{\mathrm{c}}$ & $\begin{array}{l}\text { Vestibulopathy, uveitis, headache, brainstem signs, } \\
\text { hemiparesis, cerebral venous thrombosis; } \\
\text { oral/genital ulcers }\end{array}$ & $\begin{array}{l}\text { None specifically; International Study Group } \\
\text { criteria (with pathergy test) for systemic disease }\end{array}$ \\
\hline $\begin{array}{l}\text { Neurosarcoidosis } \\
\quad[111]\end{array}$ & $\mathrm{U} ; \mathrm{RC}^{\mathrm{a}}$ & $\mathrm{F}$ & $\begin{array}{l}\text { Vestibulopathy, cranial nerve palsies, seizures, } \\
\text { aseptic meningitis, myelopathy, peripheral } \\
\text { neuropathy, pituitary dysfunction }\end{array}$ & $\begin{array}{l}\text { Contrast MRI sensitive but not specific; whole } \\
\text { body PET, biopsy involved peripheral tissue }\end{array}$ \\
\hline $\begin{array}{l}\text { Susac's syndrome } \\
\text { [112] }\end{array}$ & $\mathrm{T} ; \mathrm{C}^{\mathrm{a}, \mathrm{d}}$ & $\mathrm{T}$ & $\begin{array}{l}\text { Retinal artery occlusions; migraine, ataxia, vertigo, } \\
\text { long tract signs }\end{array}$ & $\begin{array}{l}\text { MRI (callosal ‘snowball’ lesions); retinal } \\
\text { fluoroscein angiography (multifocal distal } \\
\text { arteriolar occlusions) }\end{array}$ \\
\hline \multicolumn{5}{|l|}{ Infectious } \\
\hline $\begin{array}{l}\text { Cryptococcal } \\
\text { meningitis } \\
{[113]}\end{array}$ & $\mathrm{U} ; \mathrm{RC}^{\mathrm{a}}$ & $\mathrm{F}$ & $\begin{array}{l}\text { Headache, papilloedema, seizures, vestibulopathy, } \\
\text { cranial nerve palsies; more common in } \\
\text { immunocompromised patients }\end{array}$ & CSF Cryptococcal antigen \\
\hline $\begin{array}{l}\text { Neuroborreliosis } \\
\text { [114] }\end{array}$ & $\mathrm{U} ; \mathrm{RC}^{\mathrm{e}}$ & $\mathrm{U}^{\mathrm{f}}$ & $\begin{array}{l}\text { Lymphocytic meningitis with cranial palsies, } \\
\text { vestibulopathy }\end{array}$ & Lyme serology \\
\hline $\begin{array}{l}\text { Neurosyphilis } \\
\text { [115] }\end{array}$ & $\mathrm{U}$ & $\mathrm{T}$ & $\begin{array}{l}\text { Chorioretinitis, Argyll Robertson pupils, } \\
\text { vestibulopathy, cranial nerve palsies and brainstem } \\
\text { signs, myelopathy (tabes dorsalis), brain infarcts }\end{array}$ & Treponemal serology (blood and CSF) \\
\hline \multicolumn{5}{|l|}{ Genetic } \\
\hline CADASIL [116] & $\mathrm{U} ; \mathrm{C}^{\mathrm{a}}$ & $\mathrm{T}$ & Migraine, stroke, psychiatric disturbance & $\begin{array}{l}\text { Characteristic MRI with marked anterior } \\
\text { temporal/external capsule white matter } \\
\text { involvement Notch3 mutations }\end{array}$ \\
\hline $\begin{array}{l}\text { MELAS/other } \\
\text { mitochondrial } \\
\text { syndromes } \\
\text { [117] }\end{array}$ & $\mathrm{T} / \mathrm{F} ; \mathrm{C}$ & $\mathrm{T} / \mathrm{F}$ & $\begin{array}{l}\text { Migraine, seizures, stroke-like episodes, } \\
\text { ophthalmoplegia, myopathy, lactic acidosis, } \\
\text { diabetes mellitus }\end{array}$ & Various mitochondrial DNA mutations \\
\hline HSAN IE [118] & $\begin{array}{l}\text { T; C, } \\
\text { RC? }^{h}\end{array}$ & $\mathrm{~T}$ & $\begin{array}{l}\text { Sensory and autonomic neuropathy, optic } \\
\text { neuropathy, narcolepsy }\end{array}$ & DNMT1 mutations \\
\hline IBMPFD [119] & $\begin{array}{l}\mathrm{U} ; \\
\mathrm{RC} ?\end{array}$ & $\mathrm{~T}$ & $\begin{array}{l}\text { Frontotemporal dementia with inclusion body } \\
\text { myositis, Paget's disease of bone }\end{array}$ & $V C P$ mutations \\
\hline $\begin{array}{l}\text { Niemann-Pick } \\
\text { type C [120] }\end{array}$ & $\mathrm{F} ; \mathrm{RC}$ & $\mathrm{T}^{\mathrm{c}}$ & $\begin{array}{l}\text { Ataxia, supranuclear gaze palsy, dystonia, } \\
\text { psychiatric features, cataplexy, seizures, } \\
\text { splenomegaly }\end{array}$ & $\begin{array}{l}\text { Skin fibroblast studies (accumulation of } \\
\text { unesterified cholesterol), genotyping }\end{array}$ \\
\hline $\begin{array}{l}\text { Oculo- } \\
\text { leptomeningeal } \\
\text { amyloidosis } \\
{[121]}\end{array}$ & $\mathrm{F} ; \mathrm{RC}^{\mathrm{a}}$ & $\mathrm{F}$ & $\begin{array}{l}\text { Seizures, stroke-like episodes, headache, ataxia, } \\
\text { myelo-radiculopathy, subarachnoid haemorrhage, } \\
\text { ocular amyloid }\end{array}$ & $\begin{array}{l}\text { Abnormal meningeal enhancement on contrast } \\
\text { MRI } \\
\text { Transthyretin mutations }\end{array}$ \\
\hline $\begin{array}{l}\text { Refsum disease } \\
\text { [122] }\end{array}$ & $\mathrm{F} ; \mathrm{RC}$ & $\mathrm{U}$ & Retinitis pigmentosa, anosmia, polyneuropathy & $\begin{array}{l}\text { Raised plasma phytanic acid } \\
\text { PHYH mutation }\end{array}$ \\
\hline $\begin{array}{l}\text { Spinocerebellar } \\
\text { ataxias: [83] }\end{array}$ & $\mathrm{F} ; \mathrm{C}$ & $\mathrm{F}$ & $\begin{array}{l}\text { Truncal/limb ataxia, bulbar deficits, proprioceptive } \\
\text { impairment, neuropathy, variably prominent } \\
\text { across group }\end{array}$ & $\begin{array}{l}\text { Various mutations (most frequently, trinucleotide } \\
\text { repeat expansions) }\end{array}$ \\
\hline $\begin{array}{l}\text { Friedreich's } \\
\text { ataxia [123] }\end{array}$ & & & $\begin{array}{l}\text { Cardiomyopathy, diabetes mellitus (adult onset } \\
\text { milder) }\end{array}$ & $F X N$ expansions \\
\hline SCA13 [124] & & & $\begin{array}{l}\text { Gait/limb ataxia, dysarthria, hyperreflexia, vibration } \\
\text { sense loss }\end{array}$ & KCNC3 mutations \\
\hline $\begin{array}{l}\text { Wolfram's } \\
\text { syndrome [125] }\end{array}$ & $\mathrm{T} ; \mathrm{RC}$ & $\mathrm{F}^{\mathrm{j}}$ & Optic atrophy, diabetes & WFS1 mutations \\
\hline
\end{tabular}


Table 3 continued

\begin{tabular}{|c|c|c|c|c|}
\hline Disease & Aud & Cogn & Associated features & Diagnostic investigations \\
\hline \multicolumn{5}{|l|}{ Other } \\
\hline $\begin{array}{l}\text { Prion diseases } \\
\quad[126]\end{array}$ & $\mathrm{U} ; \mathrm{RC}$ & $\mathrm{T}$ & $\begin{array}{l}\text { Rapid neurological decline, often with prominent } \\
\text { myoclonus and ataxia }\end{array}$ & $\begin{array}{l}\text { Increased cortical/basal ganglia signal on DWI/ } \\
\text { FLAIR MRI with compatible clinical } \\
\text { phenotype; rarely prion gene mutation }(E 200 K)\end{array}$ \\
\hline $\begin{array}{l}\text { Superficial } \\
\quad \text { siderosis [127] }\end{array}$ & $\mathrm{T} ; \mathrm{RC}^{\mathrm{k}}$ & $\mathrm{F}$ & $\begin{array}{l}\text { Cerebellar ataxia, pyramidal signs, bladder } \\
\text { dysfunction, anosmia, anisocoria; may have } \\
\text { history compatible with chronic subarachnoid } \\
\text { bleeding }\end{array}$ & $\begin{array}{l}\text { Haemosiderin rimming brain/spinal cord on } \\
\text { susceptibility-weighted MRI }\end{array}$ \\
\hline
\end{tabular}

CADASIL cerebral autosomal dominant arteriopathy with subcortical infarcts and leukoencephalopathy, CJD Creutzfeldt-Jakob disease, DNMT1 DNA cytosine-5-methyltransferase 1 gene, DWI/FLAIR diffusion weighted/fluid-attenuated inversion recovery sequences, FXN frataxin gene, HSAN IE hereditary sensory and autonomic neuropathy with dementia and hearing loss type IE, IBMPFD inclusion body myositis with Paget's disease of bone and frontotemporal dementia, $K C N C 3$ potassium channel Kv3.3 gene, MELAS mitochondrial encephalopathy with lactic acidosis and stroke-like episodes, $P H Y H$ phytanoyl-CoA 2-hydroxylase gene, VCP valosin containing protein gene, WFS1 wolframin gene

The Table excludes paediatric disorders that do not also present during adult life; auditory (Aud) and cognitive (Cogn) phenotypes have been classified according to whether clinical impairments of hearing and/or cognition are: $T$ typical of the entity (a very frequent or defining feature), $F$ frequent (a common association), $U$ unusual (a recognised association). The cognitive phenotype in most cases is not diagnostic, comprising variably prominent executive, subcortical and behavioural deficits and affective changes. The auditory phenotype has been classified according to the origin of hearing loss, where (often limited) information available: $C$ cochlear, $R C$ retrocochlear (auditory nerve and/or brainstem pathways)

${ }^{\text {a }}$ May be sudden

b Generally more significant in progressive forms

c May have prominent neuropsychiatric changes

${ }^{\mathrm{d}}$ Low-to-mid-frequency loss characteristic

e May have persistent post-treatment altered hearing (e.g., loudness intolerance)

${ }^{\mathrm{f}}$ Subjective cognitive symptoms frequent

g Ménière's-like presentations may occur

h Mid-frequency loss

${ }^{\mathrm{i}}$ Auditory brainstem pathway involvement appears more functionally significant than more peripheral involvement and may disrupt temporal processing leading to deficits of spatial and speech perception (frequency of impairment varies with mutation)

${ }^{\mathrm{j}}$ May be more prominent in later disease

${ }^{\mathrm{k}}$ Primary cochlear damage may also contribute

part of a pan-modal erosion of semantic memory, linked to antero-mesial temporal lobe dysfunction [48, 53, 54]. Interestingly, in individual cases, there may be relatively preserved knowledge of melodies over environmental sounds $[55,56]$, perhaps because the abstract, nonreferential meaning systems of music have neural substrates that are separable from those mediating knowledge about the world at large (knowledge of musical instrument timbres may be affected comparably to other categories of objects). Impaired recognition of familiar voices (despite retained ability to distinguish between voices) may be a salient symptom of right temporal lobe degeneration [57, 58]: such 'associative phonagnosia' may be relatively selective for voices or accompany other deficits of person knowledge or more generalised auditory agnosia.

\section{Auditory hallucinations}

Patients with semantic dementia commonly report tinnitus (an elementary auditory hallucination), linked to structural alterations in a fronto-temporo-subcortical network [5]; while hallucinations of 'muffled' sounds or voices are often reported by patients with Lewy body dementia, frank verbal hallucinations are uncommon and generally occur as a component of more complex, multimodal hallucinations [59]. In contrast, persistent musical hallucinations (typically comprising familiar, banal tunes) are relatively commonly reported in patients with Lewy body disease and less frequently, other dementias [60]: only a minority has significant hearing loss, suggesting that aberrant cortical activity plays a key role though there may be a facilitatory effect from peripheral deafferentation [61].

\section{Abnormal auditory behaviours}

Altered emotional or 'hedonic' behavioural responses to sound are increasingly recognised in patients with dementia. Impaired processing of emotional prosody has been described in AD, Parkinson's disease, behavioural variant frontotemporal dementia, and progressive aphasia syndromes [62-65], and impaired recognition of musical and nonverbal vocal emotions in Parkinson's disease and 


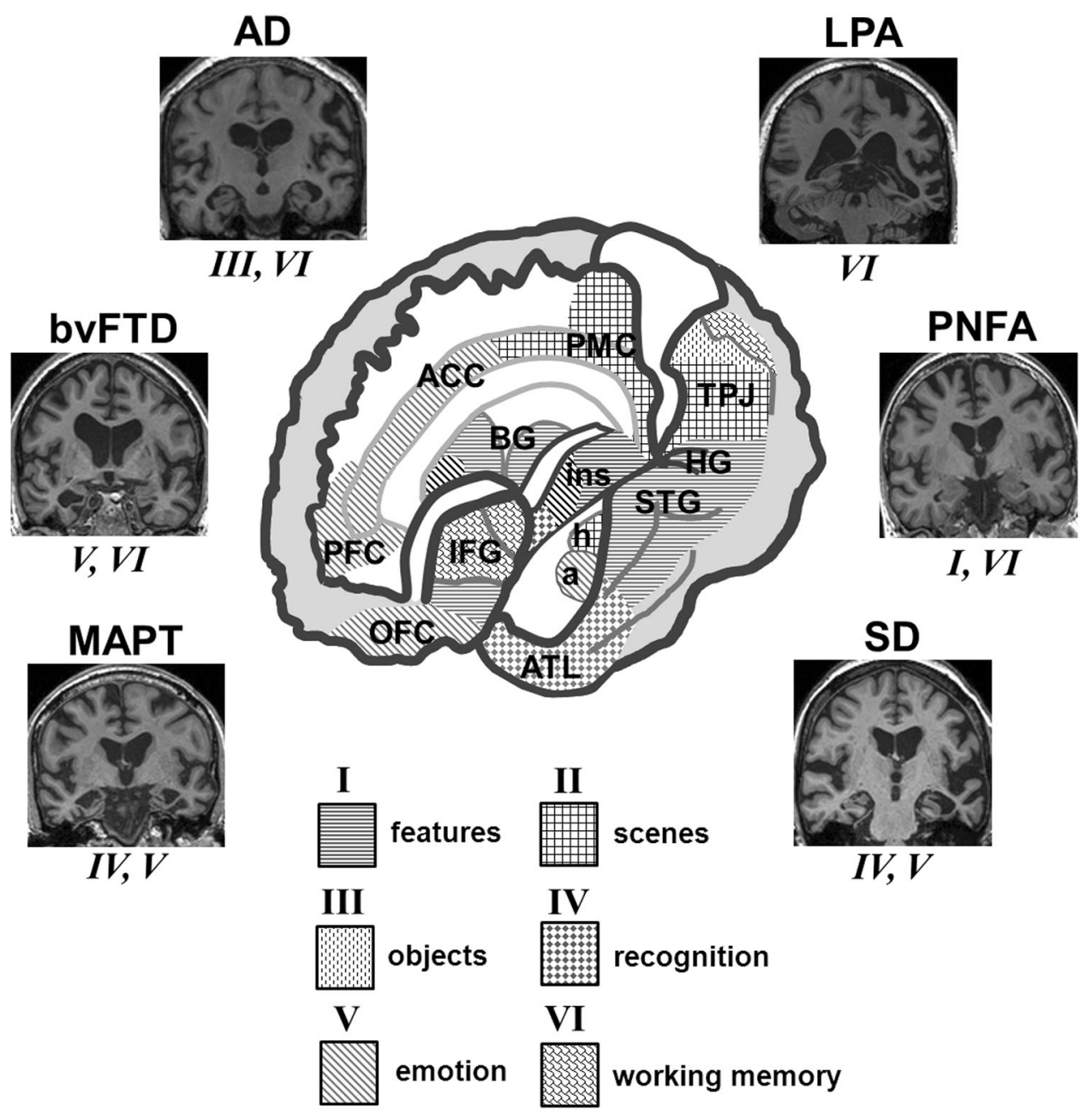

Fig. 1 Neuroanatomical signatures of disordered auditory cognition in dementias. The cutaway brain schematic (centre) shows cerebral networks that mediate key components of auditory cognition, coded $I$ to VI (below) and based on clinical and normal functional neuroanatomical evidence (see Tables 1,2); 'features' here subsumes acoustic feature detection and analysis, 'objects' corresponds to auditory apperceptive processing and 'recognition' corresponds to auditory semantic processing. The left cerebral hemisphere is projected forward in the schematic; however, neuroanatomical correlates of auditory cognition are bi-hemispherically distributed, principally, including: $a$ amygdala, $A C C$ anterior cingulate cortex, $A T L$ anterior temporal lobe, $B G$ basal ganglia, $h$ hippocampus, $H G$ Heschl's gyrus (containing primary auditory cortex), $I F G$ inferior frontal gyrus/frontal operculum, ins insula, $O F C$ orbitofrontal cortex, $P F C$ prefrontal cortex, $P M C$ posterior medial cortex (posterior

frontotemporal dementia syndromes [65, 66]. Many patients with frontotemporal dementia (and some with AD) exhibit sound aversion, while abnormal craving for music (musicophilia) is particularly associated with semantic dementia [51]; these patients may show increased sensitivity to sound (hyperacusis [5]), also described in prion disease [67]. Explicit behavioural responses may dissociate from autonomic responses to sound in dementia syndromes [68, 69]. Altered auditory hedonic behaviours in dementia have been

cingulate, precuneus), $S T G$ superior temporal gyrus/superior temporal sulcus/planum temporale, TPJ temporo-parietal junction. Side panels show characteristic profiles of regional cerebral atrophy (coronal MRI sections) and auditory cognitive functions chiefly affected in selected dementias (see also Table 2): typical Alzheimer's disease (AD), bilateral symmetrical mesial temporal and parietal lobe atrophy; behavioural variant frontotemporal dementia (bvFTD), asymmetric (predominantly right-sided) frontal and temporal lobe atrophy; logopenic aphasia (LPA) variant of Alzheimer's disease, predominantly left-sided temporo-parietal atrophy; microtubule-associated protein tau (MAPT) gene mutations, bilateral (predominantly anteromesial) temporal lobe atrophy; progressive nonfluent aphasia (PNFA), predominantly left-sided peri-Sylvian atrophy; and semantic dementia (SD), asymmetric (predominantly left-sided) anterior temporal lobe atrophy

linked to involvement of distributed cortico-subcortical circuitry that processes emotion and reward [51].

\section{A practical approach to the patient with dementia and altered hearing}

A clinical framework for assessing and managing the patient presenting with cognitive impairment and altered hearing is outlined in Table 4 and Fig. 2. 
Table 4 Taking the auditory history in patients with cognitive impairment

\begin{tabular}{|c|c|c|c|}
\hline Domain & Question & Key process probed & Significance \\
\hline \multirow[t]{2}{*}{ Background } & Previous occupation? & $\begin{array}{l}\text { Previous cognitive/ } \\
\text { auditory function, } \\
\text { noise exposure }\end{array}$ & Correct interpretation of hearing tests \\
\hline & $\begin{array}{l}\text { Previous level of musical training and interest, } \\
\text { early language development and education? }\end{array}$ & Prior auditory expertise & Correct interpretation of hearing tests \\
\hline \multirow[t]{2}{*}{ Course } & When was hearing impairment first noticed? & $\begin{array}{l}\text { Duration of impairment } \\
\text { (relative to cognitive } \\
\text { decline) }\end{array}$ & Nature of underlying disease process \\
\hline & $\begin{array}{l}\text { Has this deteriorated, fluctuated or improved } \\
\text { since onset? }\end{array}$ & Tempo of impairment & Nature of underlying disease process \\
\hline \multicolumn{4}{|l|}{ Symptoms } \\
\hline \multirow[t]{3}{*}{ Sound detection } & Is there a lack of reaction to sounds? & $\begin{array}{l}\text { Impaired sound } \\
\text { detection }\end{array}$ & May signify deafness (any cause) \\
\hline & $\begin{array}{l}\text { Is there a tendency to turn up the volume of } \\
\text { radio or TV or to ask people to speak louder? }\end{array}$ & $\begin{array}{l}\text { Impaired sound } \\
\text { detection }\end{array}$ & May signify deafness (any cause) \\
\hline & $\begin{array}{l}\text { Is there a complaint that increasing volume } \\
\text { makes sounds suddenly seem too loud? }\end{array}$ & $\begin{array}{l}\text { Impaired sound } \\
\text { detection }\end{array}$ & $\begin{array}{l}\text { May signify cochlear pathology ('loudness } \\
\text { recruitment') }\end{array}$ \\
\hline \multirow{6}{*}{$\begin{array}{l}\text { Abnormal } \\
\text { auditory } \\
\text { perception: } \\
\text { deficient }\end{array}$} & $\begin{array}{l}\text { Is there particular difficulty following } \\
\text { conversations in background noise or over a } \\
\text { noisy telephone line? }\end{array}$ & Auditory scene analysis & $\begin{array}{l}\text { May signify a cerebral disorder (e.g., } \\
\text { Alzheimer's disease) in absence of } \\
\text { significant hearing loss }\end{array}$ \\
\hline & $\begin{array}{l}\text { Is there difficulty locating sounds (e.g., an alarm } \\
\text { or mobile, a person speaking in same the } \\
\text { room)? }\end{array}$ & Auditory scene analysis & $\begin{array}{l}\text { May signify a cerebral disorder in absence } \\
\text { of significant hearing loss }\end{array}$ \\
\hline & $\begin{array}{l}\text { Is there particular difficulty understanding } \\
\text { speech versus other sounds? }\end{array}$ & Feature analysis & May signify word deafness \\
\hline & $\begin{array}{l}\text { Is there more difficulty understanding less } \\
\text { familiar accents? }\end{array}$ & Apperceptive processing & $\begin{array}{l}\text { May signify a cerebral disorder in absence } \\
\text { of significant hearing loss }\end{array}$ \\
\hline & $\begin{array}{l}\text { Is there more difficulty understanding a person's } \\
\text { tone of voice (e.g., angry or upset)? }\end{array}$ & $\begin{array}{l}\text { Apperceptive and } \\
\text { emotional processing }\end{array}$ & $\begin{array}{l}\text { May signify a frontotemporal dementia in } \\
\text { appropriate context }\end{array}$ \\
\hline & $\begin{array}{l}\text { Has there been any problem recognising } \\
\text { familiar voices, music or other sounds? }\end{array}$ & Semantic processing & $\begin{array}{l}\text { May signify auditory agnosia or semantic } \\
\text { dementia, in appropriate context }\end{array}$ \\
\hline \multirow{2}{*}{$\begin{array}{l}\text { Abnormal } \\
\text { auditory } \\
\text { perception: } \\
\text { excessive }\end{array}$} & $\begin{array}{l}\text { Is there a persistent complaint of buzzing or } \\
\text { ringing in the ears? }\end{array}$ & Tinnitus & May be peripheral or central in origin \\
\hline & $\begin{array}{l}\text { Are other sounds ever heard when no sounds are } \\
\text { present? }\end{array}$ & Formed hallucinations & $\begin{array}{l}\text { May signify Lewy body disease, in } \\
\text { appropriate context }\end{array}$ \\
\hline \multirow{2}{*}{$\begin{array}{l}\text { Abnormal } \\
\text { auditory } \\
\text { behaviour }\end{array}$} & $\begin{array}{l}\text { Is there intolerance to moderately loud sounds } \\
\text { or particular sounds? }\end{array}$ & Hyperacusis & $\begin{array}{l}\text { May signify a frontotemporal dementia in } \\
\text { appropriate context }\end{array}$ \\
\hline & $\begin{array}{l}\text { Has there been any change in liking for or } \\
\text { interest in music or other sounds? }\end{array}$ & $\begin{array}{l}\text { Auditory hedonic } \\
\text { processing }\end{array}$ & $\begin{array}{l}\text { May signify a frontotemporal dementia in } \\
\text { appropriate context }\end{array}$ \\
\hline
\end{tabular}

In all cases, a corroborating history should be sought from the patient's caregiver or other advocate. See also Tables 1, 2, 3; Fig. 2

\section{Clinical assessment}

Hearing function should be assessed in all patients receiving a diagnosis of dementia: to identify a factor that may be detracting from quality of life and impeding care [70], to gauge disability as fully as possible, and to address any reversible peripheral component. Assessment begins with a history to elicit key auditory symptoms (Table 4; Fig. 2) and systematic neurological and otological examination. Hearing impairment can easily go undetected in patients with dementia and may lead to misattribution or overestimation of cognitive compromise [71]; cognitive screening instruments that do not rely on hearing (such as the TYM test [72]) may be preferable to verbally administered tests such as the mini-mental state examination. The patient's premorbid competence in particular domains (notably, music) should be documented, and an auditory handicap questionnaire may be useful in defining the functional consequences of hearing impairment [73].

In patients with known hearing impairment, any supervening cognitive decline should be thoroughly characterised. This is particularly challenging in those with pre- 


\section{BEDSIDE}

informant history, $A H Q$, neurologic/otologic exam
INVESTIGATIONS

PTA, SiN, GIN, DLT, OAE, $A B R$, neuropsych, MRI
MANAGEMENT

$S A L T$, local services, env / behav strategies

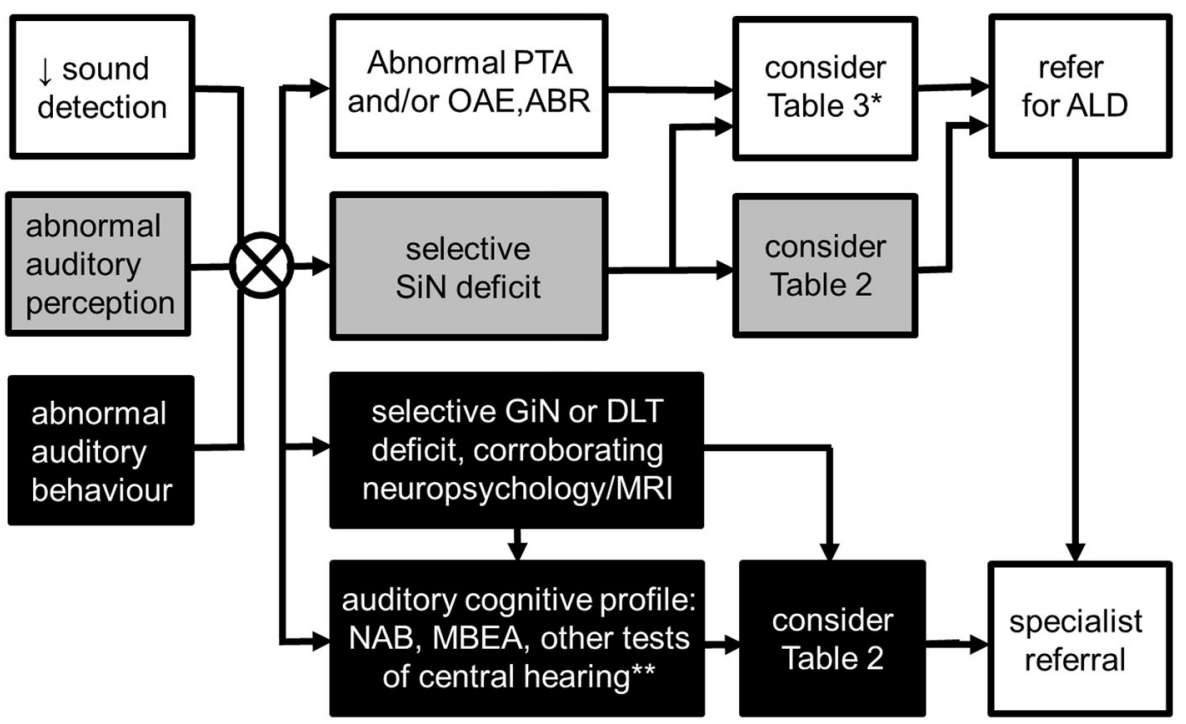

Fig. 2 A clinical approach to the patient presenting with cognitive decline and altered hearing. Our approach is based on initial thorough bedside history taking and examination to identify key auditory symptoms (see also Table 4) supplemented by investigations to characterise the nature of the patient's hearing and cognitive deficits. As clinical symptoms are rarely specific and disorders at different levels of the auditory processing hierarchy frequently coexist, we recommend a core hearing assessment battery in all cases, corroborated by general neuropsychological assessment and brain MRI. Together, these assessments often allow the patient's hearing deficit to be localised predominantly to the cochlea or ascending auditory pathways (unfilled oblongs) or to cerebral circuitry (black filled oblongs) and direct further more specific assessment for the diagnoses listed in Tables 2, 3. Other patients will have auditory deficits that are more difficult to localise or may have mixed deficits (grey oblongs); speech-in-noise perception is a useful index of real world hearing impairment but needs care in interpretation as this can be affected by pathology at different levels of the auditory system. Management in all cases should involve consideration of environmental and

lingual profound hearing loss. British Sign Language (BSL) users form a close-knit minority group defined culturally as well as linguistically; they often have difficulty in accessing appropriate assessment services and, therefore, may have more advanced dementia when diagnosed. Ideally, assessment of such individuals should be undertaken in a clinic with special expertise in working with deaf people, engaging a neuropsychologist with relevant communication skills and expertise and BSL interpreters trained in the requirements of cognitive assessment. Until recently, there were no normative data on cognition in deaf subjects; however, a cognitive screening instrument based on British Sign Language has now been developed [74]. As with any cognitive assessment, it is essential to obtain a corroborating history from an advocate or caregiver. behavioural modification strategies that optimise the patient's residual hearing function (see text) and involvement of multidisciplinary services to assess their needs and plan appropriate care delivery; we have a low threshold for a trial of hearing aids or other assistive listening devices if there is the possibility of a contributing peripheral hearing loss and in patients with more complex or central auditory deficits, onward referral to a specialist cognitive or auditory clinic may be helpful. Asterisk particularly in younger patients or where there are associated neurological or systemic features; double asterisk more specialised tests of central hearing functions if available may be useful in defining the phenotype of an auditory cortical disorder, particularly where all standard tests of hearing are unremarkable; $A B R$ auditory brainstem evoked responses, $A H Q$ auditory handicap questionnaire, $A L D$ assistive listening device, behav behavioural, env environmental, $D L T$ dichotic listening test, GiN gap-in-noise perception, $H A$ hearing aid, MBEA Montreal Battery for Evaluation of Amusia, MRI brain magnetic resonance imaging, $N A B$ Newcastle Auditory Battery, neuropsych neuropsychology, OAE otoacoustic emissions, PTA pure tone audiometry, SiN speech-in-noise perception

\section{Investigations}

Pure tone audiometry and otoacoustic emissions (supplemented by brainstem auditory evoked potentials if available) are relatively simple and well-tolerated techniques to assess cochlear and ascending auditory pathway function in cognitively impaired patients. Measurement of speech-innoise perception (Table 1) more closely reflects 'real world' hearing impairment than pure tone audiometry and (if impaired disproportionately to other indices of cochlear or ascending auditory pathway function) may provide an index of auditory cortical processing; this can be supplemented by tests such as gap-in-noise perception or dichotic listening that depend more sensitively on cortical processing of sound [75] (Table 1). If the patient or caregiver 
reports symptoms suggesting auditory cognitive dysfunction, a more comprehensive evaluation may be appropriate: age norms are available for the Newcastle Auditory Battery [76] and Montreal Battery for Evaluation of Amusia [77]. General neuropsychological assessment can identify concurrent executive or attentional deficits that may confound performance on auditory tests and may also define associated (visual and verbal) apperceptive or semantic impairments to corroborate any auditory cognitive deficit. If a complex or unusual disorder (such as auditory agnosia) is suspected, referral to a specialist clinic may be indicated. Investigation of any patient with suspected dementia should focus on identifying reversible processes and establishing the primary cause as accurately as possible (for general reviews, see [16-18]); this should include brain MRI, which may further characterise the likely neuroanatomical basis of the auditory deficit (Table 2).

\section{Management}

While effects on specific aspects of cognitive function are difficult to predict, correction of reversible hearing deficits has been shown to benefit global functioning in daily life $[20,21,23,78,79]$. Simple interventions such as earwax removal can be highly effective [79]. In addition, prescription of hearing aids and other assistive listening devices where appropriate, environmental modification strategies may be useful in managing altered hearing in patients with dementia. Examples include the use of written communication aids and electronic devices, conducting conversations face-to-face and free of significant background noise, avoiding sounds known to provoke distress, and masking techniques for musical and other auditory hallucinations. Anecdotally, cholinesterase inhibitors may benefit some patients with musical hallucinations [80]; however, there is currently little role for specific pharmacotherapy in managing auditory dysfunction in dementia. Specific auditory training protocols based on speech and nonspeech sounds have been shown to improve speech intelligibility in hearing-impaired adults [81] but have yet to be adequately assessed in dementia. Though music is unquestionably a welcome source of solace for many patients and caregivers and often a useful displacement activity, evidence for a specific role of music therapy in the management of dementia awaits bettercontrolled trials [11]. Assistive listening devices incorporating a mobile microphone are a promising strategy to compensate for deficits of auditory scene processing [73], but their utility in patients with dementia remains to be established. Management of hearing loss in dementia is an inter-disciplinary enterprise, and close collaboration with audiology, speech and language therapy, and social services is invaluable.

\section{Conclusions and future directions}

Hearing has long been the poor relation of memory and vision in the cognitive clinic. The emerging epidemiological evidence may shortly transform this situation. The comprehensive assessment of hearing in dementia presents both challenges and opportunities. There is a need to develop practical and reliable tests that can disambiguate the effects of peripheral hearing and auditory cognitive dysfunction, to develop auditory interventions directed to cognitively impaired people and to assess these systematically and longitudinally in a range of dementias, referenced to healthy older people. In addition to capturing disability and improving quality of life, a more detailed picture of the spectrum of auditory dysfunction in dementia would have considerable neurobiological and clinical resonance. Sound is a dynamic and computationally demanding sensory signal that engages complex emotional and other behaviours: the processing of sounds taxes brain networks targeted by neurodegenerative pathologies and may yet yield novel cognitive 'stress tests' for diagnosis and treatment tracking in these diseases.

\section{Compliance with ethical standards}

Conflicts of interest The Dementia Research Centre is supported by Alzheimer's Research UK, the Brain Research Trust and the Wolfson Foundation. CJDH holds an MRC PhD Studentship. CRM is funded by a Clinical Research Fellowship from the Leonard Wolfson Experimental Neurology Centre. HLG was supported by an Alzheimer Research UK PhD Fellowship. CNC is supported by The National Brain Appeal-Frontotemporal Dementia Research Fund. JDW received salary support from the Wellcome Trust (Wellcome Trust Senior Clinical Fellowship (091673/Z/10/Z). The authors report no conflicts of interest.

Study funding This work was funded by the Wellcome Trust, the UK Medical Research Council and the NIHR Queen Square Dementia Biomedical Research Unit.

Open Access This article is distributed under the terms of the Creative Commons Attribution 4.0 International License (http://creative commons.org/licenses/by/4.0/), which permits unrestricted use, distribution, and reproduction in any medium, provided you give appropriate credit to the original author(s) and the source, provide a link to the Creative Commons license, and indicate if changes were made.

\section{References}

1. Lin FR, Ferrucci L, Metter EJ et al (2011) Hearing loss and cognition in the Baltimore Longitudinal Study of aging. Neuropsychology 25:763-770. doi:10.1037/a0024238

2. Lin FR, Metter EJ, O'Brien RJ et al (2011) Hearing loss and incident dementia. Arch Neurol 68:214-220. doi:10.1001/arch neurol.2010.362.Hearing

3. Lin F, Ferrucci L, An Y et al (2014) Association of hearing impairment with brain volume changes in older adults. Neuroimage 90:84-92. doi:10.1016/j.neuroimage.2013.12.059 
4. Johnson J, Chow M (2015) Hearing and music in dementia. Handb Clin Neurol. doi:10.14440/jbm.2015.54.A

5. Mahoney CJ, Rohrer JD, Goll JC et al (2011) Structural neuroanatomy of tinnitus and hyperacusis in semantic dementia. J Neurol Neurosurg Psychiatry 82:1274-1278. doi:10.1136/jnnp. 2010.235473

6. Goll JC, Kim LG, Ridgway GR et al (2012) Impairments of auditory scene analysis in Alzheimer's disease. Brain 135:190-200. doi:10.1093/brain/awr260

7. Goll JC, Kim LG, Hailstone JC et al (2011) Auditory object cognition in dementia. Neuropsychologia 49:2755-2765. doi:10. 1016/j.neuropsychologia.2011.06.004

8. Hailstone JC, Ridgway GR, Bartlett JW et al (2012) Accent processing in dementia. Neuropsychologia 50:2233-2244. doi:10.1016/j.neuropsychologia.2012.05.027

9. Golden HL, Nicholas JM, Yong KXX et al (2015) Auditory spatial processing in Alzheimer's disease. Brain 138:189-202. doi:10.1093/brain/awu337

10. Golden HL, Agustus JL, Goll JC et al (2015) Functional neuroanatomy of auditory scene analysis in Alzheimer's disease. Neuroimage Clin 7:699-708. doi:10.1016/j.nicl.2015.02.019

11. Vink AC, Birks JS, Bruinsma MS, Scholten RJS (2004) Music therapy for people with dementia. Cochrane database Syst Rev. doi:10.1002/14651858.CD003477.pub2

12. Cope TE, Baguley DM, Griffiths TD (2015) The functional anatomy of central auditory processing. Pract Neurol 15:302-308. doi:10.1136/practneurol-2014-001073

13. Goll JC, Crutch SJ, Warren JD (2010) Central auditory disorders: toward a neuropsychology of auditory objects. Curr Opin Neurol 23:617-627. doi:10.1097/WCO.0b013e32834027f6

14. Griffiths TD, Warren JD (2004) What is an auditory object? Nat Rev Neurosci 5:887-892. doi:10.1038/nrn1538

15. Prince M, Knapp M, Guerchet M, McCrone P, Prina M, ComasHerrera M et al (2014) Dementia UK: update. Alzheimer's Society, $136 \mathrm{p}$

16. Warren JD, Rohrer JD, Rossor MN (2013) Frontotemporal dementia. BMJ 4827:1-9. doi:10.1136/bmj.f4827

17. Rossor MN, Fox NC, Mummery CJ et al (2010) The diagnosis of young-onset dementia. Lancet Neurol 9:793-806. doi:10. 1016/S1474-4422(10)70159-9

18. Warren JD, Fletcher PD, Golden HL (2012) The paradox of syndromic diversity in Alzheimer disease. Nat Rev Neurol 8:451-464. doi:10.1038/nrneurol.2012.135

19. Gates GA, Mills JH (2005) Presbycusis. Lancet 366:1111-1120. doi:10.1016/S0140-6736(05)67423-5

20. Panza F, Solfrizzi V, Seripa D et al (2015) Age-related hearing impairment and frailty in Alzheimer's disease: interconnected associations and mechanisms. Front Aging Neurosci 7:113. doi:10.3389/fnagi.2015.00113

21. Schmulian Taljaard D, Olaithe M, Brennan-Jones CG et al (2015) The relationship between hearing impairment and cognitive function: a meta-analysis in adults. Clin Otolaryngol. doi:10.1111/coa.12607

22. Uhlmann R, Larson E, Rees $T$ et al (1989) Relationship of hearing impairment to dementia and cognitive dysfunction in older adults. JAMA 261:1916-1919

23. Dawes P, Emsley R, Cruickshanks KJ et al (2015) Hearing loss and cognition: the role of hearing aids, social isolation and depression. PLoS One 10:1-9. doi:10.1371/journal.pone.0119616

24. Peelle JE, Troiani V, Grossman M, Wingfield A (2011) Hearing loss in older adults affects neural systems supporting speech comprehension. J Neurosci 31:12638-12643. doi:10.1523/ JNEUROSCI.2559-11.2011

25. Cardin V (2016) Effects of aging and adult-onset hearing loss on cortical auditory regions. Front Neurosci 10:00199. doi:10.3389/ fnins.2016.00199
26. Sinha UK, Hollen KM, Rodriguez R, Miller CA (1993) Auditory system degeneration in Alzheimer's disease. Neurology 43:779-785

27. Parvizi J, Van Hoesen GW, Damasio A (2001) The selective vulnerability of brainstem nuclei to Alzheimer's disease. Ann Neurol 49:53-66. doi:10.1002/1531-8249(200101)49:1<53:AIDANA30 $>3.0 . \mathrm{CO} ; 2-\mathrm{Q}$

28. Liu L, Shen $\mathrm{P}, \mathrm{He} \mathrm{T}$ et al (2016) Noise induced hearing loss impairs spatial learning/memory and hippocampal neurogenesis in mice. Sci Rep 6:20374. doi:10.1038/srep20374

29. Park SY, Kim MJ, Sikandaner H et al (2016) A causal relationship between hearing loss and cognitive impairment. Acta Otolaryngol 136:480-483. doi:10.3109/00016489.2015.1130857

30. Strouse AL, Hall JW, Burger MC (1995) Central auditory processing in Alzheimer's disease. Ear Hear 16:230-238

31. Gates GA, Cobb JL, Linn RT et al (1996) Central auditory dysfunction, cognitive dysfunction, and dementia in older people. Arch Otolaryngol Head Neck Surg 122:161-167

32. Idrizbegovic E, Hederstierna C, Dahlquist M et al (2011) Central auditory function in early Alzheimer's disease and in mild cognitive impairment. Age Ageing 40:249-254. doi:10.1093/ ageing/afq168

33. Quaranta N, Coppola F, Casulli M et al (2014) The prevalence of peripheral and central hearing impairment and its relation to cognition in older adults. Audiol Neurootol 19(Suppl 1):10-14. doi:10.1159/000371597

34. Gates GA, Anderson ML, McCurry SM et al (2011) Central auditory dysfunction as a harbinger of Alzheimer dementia. Arch Otolaryngol Head Neck Surg 137:390-395. doi:10.1001/ archoto.2011.28

35. Golob EJ, Ringman JM, Irimajiri R et al (2009) Cortical eventrelated potentials in preclinical familial Alzheimer disease. Neurology 73:1649-1655. doi:10.1212/WNL.0b013e3181c1de77

36. Gold M, Lightfoot LA, Hnath-Chisolm T et al (1996) Hearing loss in a memory disorders clinic. Arch Neurol 53:922. doi:10. 1001/archneur.1996.00550090134019

37. Vitale C, Marcelli V, Allocca R et al (2012) Hearing impairment in Parkinson's disease: expanding the nonmotor phenotype. Mov Disord 27:1530-1535. doi:10.1002/mds. 25149

38. Baloyannis SJ, Manolides SL, Manolides LS (2011) Dendritic and spinal pathology in the acoustic cortex in Alzheimer's disease: morphological estimation in Golgi technique and electron microscopy. Acta Otolaryngol 131:610-612. doi:10.3109/ 00016489.2010 .539626

39. Baloyannis SJ, Mauroudis I, Manolides SL, Manolides LS (2011) The acoustic cortex in frontotemporal dementia: a Golgi and electron microscope study. Acta Otolaryngol 131:359-361. doi:10.3109/00016489.2010.539267

40. Baloyannis SJ, Manolidis SL, Manolidis LS (1995) Synaptic alterations in acoustic cortex in Creutzfeldt-Jacob disease. Acta Otolaryngol 115:202-205

41. Baloyannis SJ (2005) The acoustic cortex in vascular dementia: a Golgi and electron microscope study. J Neurol Sci 229-230:51-55. doi:10.1016/j.jns.2004.11.025

42. Clark CN, Warren JD (2016) Emotional caricatures in frontotemporal dementia. Cortex 76:134-136. doi:10.1016/j.cortex. 2015.07.026

43. Orimo S, Ozawa E, Uematsu M et al (2000) A case of Creutzfeldt-Jakob disease presenting with auditory agnosia as an initial manifestation. Eur Neurol 44:256-258. doi:10.1159/ 000008250

44. Krishna P, Bauer C (2004) Hearing loss as the initial presentation of Creutzfeldt-Jakob disease. Ear Nose Throat J 83:535, 538, 540 passim

45. Otsuki M, Soma Y, Sato M et al (1998) Slowly progressive pure word deafness. Eur Neurol 951:135-140 
46. Iizuka O, Suzuki K, Endo K (2007) Pure word deafness and pure anarthria in a patient with frontotemporal dementia. Eur $\mathrm{J}$ Neurol 14:473-475. doi:10.1111/j.1468-1331.2006.01671.x

47. Kuramoto S, Hirano T, Uyama E et al (2002) A case of slowly progressive aphasia accompanied with auditory agnosia. Rinsho Shinkeigaku Clin Neurol 42:299-303

48. Goll JC, Crutch SJ, Loo JHY et al (2010) Non-verbal sound processing in the primary progressive aphasias. Brain 133:272-285. doi:10.1093/brain/awp235

49. Grube M, Bruffaerts R, Schaeverbeke J et al (2016) Core auditory processing deficits in primary progressive aphasia. Brain 139:1817-1829. doi:10.1093/brain/aww067

50. Uttner I, Mottaghy FM, Schreiber H et al (2006) Primary progressive aphasia accompanied by environmental sound agnosia: a neuropsychological, MRI and PET study. Psychiatry Res 146:191-197. doi:10.1016/j.pscychresns.2005.12.003

51. Fletcher PD, Downey LE, Golden HL et al (2015) Auditory hedonic phenotypes in dementia: a behavioural and neuroanatomical analysis. Cortex 67:95-105. doi:10.1016/j.cortex. 2015.03.021

52. Fletcher PD, Downey LE, Agustus JL et al (2013) Agnosia for accents in primary progressive aphasia. Neuropsychologia 51:1709-1715. doi:10.1016/j.neuropsychologia.2013.05.013

53. Bozeat S, Lambon Ralph MA, Patterson K et al (2000) Nonverbal semantic impairment in semantic dementia. Neuropsychologia 38:1207-1215. doi:10.1016/S0028-3932(00)00034-8

54. Golden HL, Downey LE, Fletcher PD et al (2015) Identification of environmental sounds and melodies in syndromes of anterior temporal lobe degeneration. J Neurol Sci 352:94-98. doi:10. 1016/j.jns.2015.03.007

55. Omar R, Hailstone JC, Warren JE et al (2010) The cognitive organization of music knowledge: a clinical analysis. Brain 133:1200-1213. doi:10.1093/brain/awp345

56. Hsieh S, Hornberger M, Piguet O, Hodges JR (2011) Neural basis of music knowledge: evidence from the dementias. Brain 134:2523-2534. doi:10.1093/brain/awr190

57. Hailstone JC, Ridgway GR, Bartlett JW et al (2011) Voice processing in dementia: a neuropsychological and neuroanatomical analysis. Brain 134:2535-2547. doi:10.1093/brain/ awr205

58. Hailstone JC, Crutch SJ, Vestergaard MD et al (2010) Progressive associative phonagnosia: a neuropsychological analysis. Neuropsychologia 48:1104-1114. doi:10.1016/j.neu ropsychologia.2009.12.011

59. Holroyd S, Currie L, Wooten GF (2001) Prospective study of hallucinations and delusions in Parkinson's disease. J Neurol Neurosurg Psychiatry 70:734-738

60. Golden EC, Josephs KA (2015) Minds on replay: musical hallucinations and their relationship to neurological disease. Brain 138:3793-3802. doi:10.1093/brain/awv286

61. Griffiths TD (2000) Musical hallucinosis in acquired deafness: phenomenology and brain substrate. Brain 123:2065-2076. doi:10.1093/brain/123.10.2065

62. Horley K, Reid A, Burnham D (2010) Emotional prosody perception and production in dementia of the Alzheimer's type. J Speech Lang Hear Res 53:1132-1146. doi:10.1044/10924388(2010/09-0030)

63. Dara C, Kirsch-Darrow L, Ochfeld E et al (2013) Impaired emotion processing from vocal and facial cues in frontotemporal dementia compared to right hemisphere stroke. Neurocase 19:521-529. doi:10.1080/13554794.2012.701641

64. Rohrer JD, Sauter D, Scott S et al (2012) Receptive prosody in nonfluent primary progressive aphasias. Cortex 48:308-316. doi:10.1016/j.cortex.2010.09.004

65. Lima CF, Garrett C, Castro SL (2013) Not all sounds sound the same: Parkinson's disease affects differently emotion processing in music and in speech prosody. J Clin Exp Neuropsychol 35:373-392. doi:10.1080/13803395.2013.776518

66. Omar R, Henley SMD, Bartlett JW et al (2011) The structural neuroanatomy of music emotion recognition: evidence from frontotemporal lobar degeneration. Neuroimage 56:1814-1821. doi:10.1016/j.neuroimage.2011.03.002

67. Merkler AE, Prasad M, Lavi E, Safdieh J (2014) Hyperacusis as the initial presentation of Creutzfeldt-Jakob disease. Neurol Neuroimmunol Neuroinflamm 1:e32. doi:10.1212/NXI. 0000000000000032

68. Fletcher PD, Nicholas JM, Shakespeare TJ et al (2015) Physiological phenotyping of dementias using emotional sounds. Alzheimer's Dement Diagn Assess Dis Monit 1:170-178. doi:10.1016/j.dadm.2015.02.003

69. Fletcher PD, Nicholas JM, Shakespeare TJ et al (2015) Dementias show differential physiological responses to salient sounds. Front Behav Neurosci 9:73. doi:10.3389/fnbeh.2015. 00073

70. Weinstein BE, Ventry IM (1982) Hearing impairment and social isolation in the elderly. J Speech Hear Res 25:593-599. doi:10. 1044 /jshr.2504.593

71. Weinstein B (1986) Hearing loss and senile dementia in the institutionalized elderly. Clin Gerontol 4:3-15. doi:10.1300/ J018v04n03_02

72. Brown J, Pengas G, Dawson K, Brown LA, Clatworthy P (2009) Self administered cognitive screening test (TYM) for detection of Alzheimer's disease: cross sectional study. BMJ 338:b2030. doi:10.1136/bmj.b2030

73. Martin JS, Jerger JF (2005) Some effects of aging on central auditory processing. J Rehabil Res Dev 42:25-44. doi:10.1682/ JRRD.2004.12.0164

74. Atkinson J, Denmark T, Marshall J et al (2015) Detecting cognitive impairment and dementia in deaf people: the British Sign Language cognitive screening test. Arch Clin Neuropsychol 30:694-711. doi:10.1093/arclin/acv042

75. Musiek FE, Chermak GD (2015) Psychophysical and behavioral peripheral and central auditory tests. Handb Clin Neurol 129:313-332. doi:10.1016/B978-0-444-62630-1.00018-4

76. Griffiths TD, Dean JL, Woods W et al (2001) The Newcastle Auditory Battery (NAB). A temporal and spatial test battery for use on adult naive subjects. Hear Res 154:165-169

77. Peretz I, Champod AS, Hyde K (2003) Varieties of musical disorders: the Montreal Battery of evaluation of amusia. Ann N Y Acad Sci 999:58-75. doi:10.1196/annals.1284.006

78. Allen NH, Burns A, Newton V et al (2003) The effects of improving hearing in dementia. Age Ageing 32:189-193

79. Sugiura S, Yasue M, Sakurai T et al (2014) Effect of cerumen impaction on hearing and cognitive functions in Japanese older adults with cognitive impairment. Geriatr Gerontol Int 14(Suppl 2):56-61. doi:10.1111/ggi.12251

80. Blom JD, Adriaan J, Coebergh F et al (2015) Musical hallucinations treated with acetylcholinesterase inhibitors. Front Psychiatry. doi: $10.3389 /$ fpsyt.2015.00046

81. Henshaw H, Ferguson MA (2013) Efficacy of individual computer-based auditory training for people with hearing loss: a systematic review of the evidence. PLoS One 8:e62836. doi:10. 1371/journal.pone.0062836

82. Clark CN, Golden HL, Warren JD (2015) Acquired amusia. Handb Clin Neurol 129:607-631. doi:10.1016/B978-0-44462630-1.00034-2

83. Zeigelboim BS, de Carvalho HAS, Teive HAG et al (2015) Central auditory processing in patients with spinocerebellar ataxia. Hear Res 327:235-244. doi:10.1016/j.heares.2015.07.006

84. Kay J, Lesser R, Coltheart M (1992) Psycholinguistic assessments of language processing in aphasia. Lawrence Erlbaum Associates, Hove 
85. Johnson J, Chang C, Brambati S et al (2011) Music recognition in frontotemporal lobar degeneration and Alzheimer disease. Cogn Behav Neurol 24:74-84. doi:10.1097/WNN.0b013e31821 de326.Music

86. Hsieh S, Hornberger M, Piguet O, Hodges JR (2012) Brain correlates of musical and facial emotion recognition: evidence from the dementias. Neuropsychologia 50:1814-1822. doi:10. 1016/j.neuropsychologia.2012.04.006

87. Brønnick KS, Nordby H, Larsen JP, Aarsland D (2010) Disturbance of automatic auditory change detection in dementia associated with Parkinson's disease: a mismatch negativity study. Neurobiol Aging 31:104-113. doi:10.1016/j.neurobiola ging.2008.02.021

88. Cheng C-H, Wang P-N, Hsu W-Y, Lin Y-Y (2012) Inadequate inhibition of redundant auditory inputs in Alzheimer's disease: an MEG study. Biol Psychol 89:365-373. doi:10.1016/j.biopsy cho.2011.11.010

89. Grimes AM, Grady CL, Foster NL et al (1985) Central auditory function in Alzheimer's disease. Neurology 35:352-358

90. Grady CL, Grimes AM, Patronas N et al (1989) Divided attention, as measured by dichotic speech performance, in dementia of the Alzheimer type. Arch Neurol 46:317-320

91. Eustache F, Lambert J, Cassier C et al (1995) Disorders of auditory identification in dementia of the Alzheimer type. Cortex 31:119-127. doi:10.1016/S0010-9452(13)80110-4

92. Brandt J, Bakker A, Maroof DA (2010) Auditory confrontation naming in Alzheimer's disease. Clin Neuropsychol 24:1326-1338. doi:10.1080/13854046.2010.518977

93. Rapcsak SZ, Kentros M, Rubens AB (1989) Impaired recognition of meaningful sounds in Alzheimer's disease. Arch Neurol 46:1298-1300

94. Bouma A, Gootjes L (2011) Effects of attention on dichotic listening in elderly and patients with dementia of the Alzheimer type. Brain Cogn 76:286-293. doi:10.1016/j.bandc.2011.02.008

95. Grahn JA, Brett M (2009) Impairment of beat-based rhythm discrimination in Parkinson's disease. Cortex 45:54-61. doi:10. 1016/j.cortex.2008.01.005

96. Hughes LE, Rowe JB (2013) The impact of neurodegeneration on network connectivity: a study of change detection in frontotemporal dementia. J Cogn Neurosci 25:802-813. doi:10. 1162/jocn_a_00356

97. Agustus JL, Mahoney CJ, Downey LE et al (2015) Functional MRI of music emotion processing in frontotemporal dementia. Ann N Y Acad Sci 1337:232-240. doi:10.1111/nyas.12620

98. Goll JC, Ridgway GR, Crutch SJ et al (2012) Nonverbal sound processing in semantic dementia: a functional MRI study. Neuroimage 61:170-180. doi:10.1016/j.neuroimage.2012.02. 045

99. Hardy CJD, Buckley AH, Downey LE et al (2015) The language profile of behavioral variant frontotemporal dementia. J Alzheimers Dis 50:359-371. doi:10.3233/JAD-150806

100. Kertesz A, Ang LC, Jesso S et al (2013) Psychosis and hallucinations in frontotemporal dementia with the C9ORF72 mutation: a detailed clinical cohort. Cogn Behav Neurol 26:146-154. doi:10.1097/WNN.0000000000000008

101. Rohrer JD, Crutch SJ, Warrington EK, Warren JD (2010) Progranulin-associated primary progressive aphasia: a distinct phenotype? Neuropsychologia 48:288-297. doi:10.1016/j.neu ropsychologia.2009.09.017

102. Rohrer JD, Warren JD, Barnes J et al (2008) Mapping the progression of progranulin-associated frontotemporal lobar degeneration. Nat Clin Pract Neurol 4:455-460. doi:10.1038/ ncpneuro0869

103. Chow ML, Brambati SM, Gorno-Tempini ML et al (2010) Sound naming in neurodegenerative disease. Brain Cogn 72:423-429. doi:10.1016/j.bandc.2009.12.003
104. Ghosh BCP, Calder AJ, Peers PV et al (2012) Social cognitive deficits and their neural correlates in progressive supranuclear palsy. Brain 135:2089-2102. doi:10.1093/brain/aws128

105. Saft C, Schüttke A, Beste C et al (2008) fMRI reveals altered auditory processing in manifest and premanifest Huntington's disease. Neuropsychologia 46:1279-1289. doi:10.1016/j.neu ropsychologia.2007.12.002

106. Cheng C-H, Soong B-W, Hsu W-Y, Lin Y-Y (2014) Reduced automatic frontal response to auditory deviance in Huntington's disease as indexed by magnetic mismatch negativity. J Clin Neurosci 21:1773-1778. doi:10.1016/j.jocn.2014.01.019

107. Cope TE, Grube M, Singh B et al (2014) The basal ganglia in perceptual timing: timing performance in Multiple System Atrophy and Huntington's disease. Neuropsychologia 52:73-81. doi:10.1016/j.neuropsychologia.2013.09.039

108. Lampropoulos CE, Hughes GR (2004) The antiphospholipid (Hughes') syndrome: changing the face of neurology. Eur J Intern Med 15:147-150. doi:10.1016/j.ejim.2004.01.017

109. Furst M, Levine RA (2015) Hearing disorders in multiple sclerosis. Handb Clin Neurol 129:649-665. doi:10.1016/B9780-444-62630-1.00036-6

110. Gemignani G, Berrettini S, Bruschini P et al (1991) Hearing and vestibular disturbances in Behçet's syndrome. Ann Otol Rhinol Laryngol 100:459-463

111. Johnson PB, Melbourne-Chambers R, Saindane AM et al (2014) A Case of Neurosarcoidosis with Labyrinthine Involvement. Case Rep Radiol 2014:1-5. doi:10.1155/2014/530431

112. Yurtsever B, Çabalar M, Kaya $\mathrm{H}$ et al (2015) A rare cause of hearing loss: Susac syndrome. J Int Adv Otol 11:167-169. doi:10.5152/iao.2015.1002

113. Maslan MJ, Graham MD, Flood LM (1985) Cryptococcal meningitis: presentation as sudden deafness. Am J Otol 6:435-437

114. Shotland LI, Mastrioanni MA, Choo DL et al (2003) Audiologic manifestations of patients with post-treatment Lyme disease syndrome. Ear Hear 24:508-517. doi:10.1097/01.AUD. 0000100205.25774.5F

115. Cassilde AL, Barnaud G, Baccar S et al (2009) Neurosyphilis presenting with dementia, chronic chorioretinitis and adverse reactions to treatment: a case report. Eur Ann Otorhinolaryngol Head Neck Dis 131:389-391. doi:10.1016/j.anorl.2014.02.003

116. Phillips JS, King JA, Chandran S et al (2005) Cerebral autosomal dominant arteriopathy with subcortical infarcts and leukoencephalopathy (CADASIL) presenting with sudden sensorineural hearing loss. J Laryngol Otol 119:148-151. doi:10. $1258 / 0022215053419880$

117. Chinnery PF, Elliott C, Green GR et al (2000) The spectrum of hearing loss due to mitochondrial DNA defects. Brain 123:82-92. doi:10.1093/brain/123.1.82

118. Baets J, Duan X, Wu Y et al (2015) Defects of mutant DNMT1 are linked to a spectrum of neurological disorders. Brain 138:845-861. doi:10.1093/brain/awv010

119. Djamshidian A, Schaefer J, Haubenberger D et al (2009) A novel mutation in the VCP gene (G157R) in a German family with inclusion-body myopathy with Paget disease of bone and frontotemporal dementia. Muscle Nerve 39:389-391. doi:10. $1002 /$ mus. 21225

120. King KA, Gordon-Salant S, Yanjanin N et al (2013) Auditory phenotype of Niemann-Pick disease, type C1. Ear Hear 35:110-117. doi:10.1097/AUD.0b013e3182a362b8

121. Blevins G, Macaulay R, Harder S et al (2003) Oculoleptomeningeal amyloidosis in a large kindred with a new transthyretin variant Tyr69His. Neurology 60:1625-1630

122. Bamiou D-E, Spraggs PRD, Gibberd FB et al (2003) Hearing loss in adult Refsum's disease. Clin Otolaryngol Allied Sci 28:227-230 
123. Rance G, Fava R, Baldock H et al (2008) Speech perception ability in individuals with Friedreich ataxia. Brain 131:2002-2012. doi:10.1093/brain/awn104

124. Middlebrooks JC, Nick HS, Subramony SH et al (2013) Mutation in the kv3.3 voltage-gated potassium channel causing spinocerebellar ataxia 13 disrupts sound-localization mechanisms. PLoS One 8:e76749. doi:10.1371/journal.pone.0076749

125. Santarelli R (2010) Information from cochlear potentials and genetic mutations helps localize the lesion site in auditory neuropathy. Genome Med 2:91. doi:10.1186/gm212
126. Salazar R, Cerghet M, Ramachandran V (2014) Bilateral hearing loss heralding sporadic Creutzfeldt-Jakob disease: a case report and literature review. Otol Neurotol 35:1327-1329. doi:10.1097/MAO.0000000000000485

127. Fearnley JM, Stevens JM, Rudge P (1995) Superficial siderosis of the central nervous system. Brain 118:1051-1066. doi:10. 1093/brain/118.4.1051 